\section{In memoriam of Prof. Giovanni Mazzotti}

Prof. Giovanni Mazzotti, Fellow of the Italian Society for Histochemistry passed away in June. He was a good friend, a charming teacher, and a wise and kind man.

Giovanni Mazzotti received his degree in Biological Sciences in 1971 and his medical degree in 1978 at the University of Bologna. He served as Assistant Professor in Human Anatomy from 1974 to 1980 at the University of Bologna. During this period, he established the basis of an advanced approach for the study of fine morphological modifications in immunocompetent organs induced by ionizing radiation, an approach that anticipated some aspects of his future investigations. In fact, since 1978, following the recruitment of Francesco Antonio Manzoli to the Chair of Human Anatomy at the University of Bologna, Giovanni Mazzotti was part of a motivated research group involved in the characterization of a signaling pathway in the nucleus, a system independent with respect to that at the plasma membrane.

In 1980 Giovanni Mazzotti became Professor of Human Anatomy and, from 1980 to 1984, Director of the Institute of Normal Human Morphology at the University of Chieti; during this period he was responsible for the organizing activity of the Institute, which, under the guide of Prof. Manzoli, Dean of the Faculty, was attaining a prominent role within the Uni versity of Chieti.

In 1984 he came back to the University of Bologna, as Full Professor of Human Anatomy at the School of Dentistry until 1994, and then at the School of Medicine from 1995. In these years, his scientific activity was dedicated to three main subjects: fine organization of the cell nucleus, chromatin and chromosomes, dynamic detection of DNA replication and intracellular localization of specific phospholipids involved in signal transduction. In each of these topics, his approach was based on innovative, frequently original, techniques, such as quantitative autoradiography, immune-gold labeling, as well as a combined use of transmission and high resolution transmission electron microscopy (FEISEM). Much of his experimental results, originally interpreted and discussed in more than one hundred original papers, still remain valid and contribute to an advanced interpretation of key events linking the fine organization of nuclear components to their molecular activity.

Recently, his scientific interest extended to some aspects of the interactions of biocompatible materials with mineralized tissues, mainly with dentine. Also in this clinical field, his use of advanced techniques of specific labeling associated to high resolution EM provided new insight into the field of cellular toxicity of molecular species released by biomaterials.

Giovanni Mazzotti's scientific accomplishment led him to be elected Fellow at the Academy of Sciences of Bologna, and from 1996 to 1999, Member of the Scientific Council of the Istituto Superiore di Sanità (ISS). He was also appointed as Vice-President of the Italian Society for Histochemistry from 2002 to 2005.

Besides his excellent scientific work, Giovanni Mazzotti was naturally and progressively more and more engaged in teaching Human Anatomy. Doing this with devotion, he clearly realized that many Italian medical schools were facing a crisis in anatomical education, mainly due to the shortage of both dissection facilities and trained and qualified teams teaching gross anatomy in a clinically oriented way. To overcome this lack, Giovanni Mazzotti, with a selected group of students, attended gross anatomy summer courses at the New York University, in order to create a staff of senior students which can help firstyear students in learning gross anatomy at the dissection table. He also took the trouble of obtaining the funds to provide the dissection room at the Institute of Anatomy with the required safety facilities; the new dissection room will opened next academic year; unfortunately Giovanni will not be able to finally see his wishes fulfilled.

The engagement of Giovanni Mazzotti in the academic life is highlighted by many appointments: from 1991 to 1994 President of the Corso di Laurea (PhD Programme) of Dentistry of the Faculty of Medicine, University of Bologna; from 2006 to 2009 Member of the Academic Senate of the University of Bologna; from 2006 to 2009 President of the European Federation for Experimental Morphology (EFEM). Giovanni Mazzotti was also appointed, until his death, as Member of the Council of the Dean of the Faculty of Medicine of the University of Bologna, President of the Corso di Laurea (MD

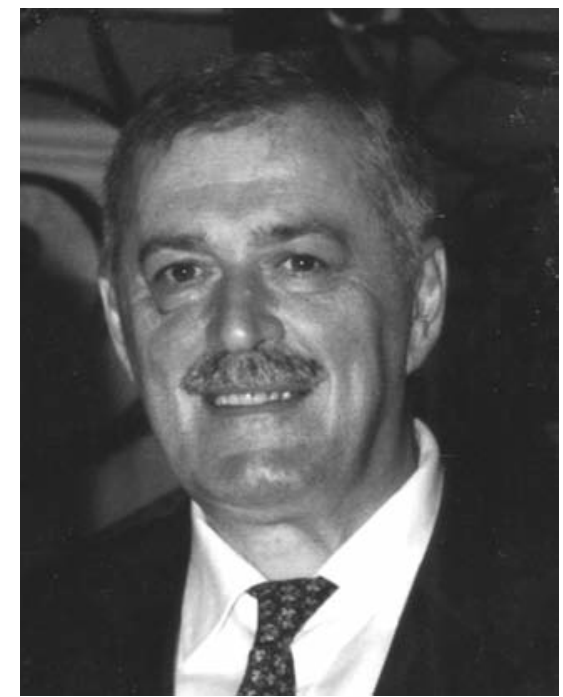

Programme) of Medicine of the University of Bologna and Italian Consultant of the International Convention of New York University with the University of Bologna for the teaching of Human Anatomy. Besides this impressive involvement in academic life, Giovanni Mazzotti was also appointed as President of the Provincial Committee of Bologna of the Red Cross of Italy, thus contributing to social initiatives in several countries.

Giovanni Mazzotti was one of us, a member of our team, a friend and a wise counselor; he was also a pleasant and fascinating gentleman who loved life, friendship, culture and, most of all, his family and his students. Even during these difficult last three years, Gianni maintained his optimistic behavior, at least with all of us, in order to relieve from our shoulders the load of an irreversible destiny.

In remembering Giovanni Mazzotti, the whole community of Italian morphologists celebrate his contribution to the development of the teaching of Human Anatomy and his scientific flair. We, as friends, will try to fulfill his legacy.

Francesco Antonio Manzoli

Nadir M. Maraldi

Silvano Capitani

Lucio Cocco 